NBER WORKING PAPER SERIES

\title{
UNPACKING NEIGHBORHOOD INFLUENCES ON EDUCATION OUTCOMES: SETTING THE STAGE FOR FUTURE RESEARCH
}

\author{
David J. Harding \\ Lisa Gennetian \\ Christopher Winship \\ Lisa Sanbonmatsu \\ Jeffrey R. Kling \\ Working Paper 16055 \\ http://www.nber.org/papers/w16055 \\ NATIONAL BUREAU OF ECONOMIC RESEARCH \\ 1050 Massachusetts Avenue \\ Cambridge, MA 02138 \\ June 2010
}

The views expressed here are those of the authors and should not be interpreted as those of the Congressional Budget Office or the National Bureau of Economic Research. We thank Greg Duncan, Richard Murnane, Rebecca Blank, Robert Sampson, Steve Raudenbush, Patrick Sharkey, Susan Clampet-Lundquist, Tama Leventhal, Larry Katz, Robert Moffitt, and participants at the Social Inequality and Educational Disadvantage conferences and the University of Michigan Robert Wood Johnson Health Policy Seminar for helpful comments on previous versions of this paper. This paper was prepared for Social Inequality and Educational Disadvantage, edited by Greg Duncan and Richard Murnane, Russell Sage Foundation Press.

NBER working papers are circulated for discussion and comment purposes. They have not been peerreviewed or been subject to the review by the NBER Board of Directors that accompanies official NBER publications.

(C) 2010 by David J. Harding, Lisa Gennetian, Christopher Winship, Lisa Sanbonmatsu, and Jeffrey R. Kling. All rights reserved. Short sections of text, not to exceed two paragraphs, may be quoted without explicit permission provided that full credit, including $\odot$ notice, is given to the source. 
Unpacking Neighborhood Influences on Education Outcomes: Setting the Stage for Future Research

David J. Harding, Lisa Gennetian, Christopher Winship, Lisa Sanbonmatsu, and Jeffrey R. Kling NBER Working Paper No. 16055

June 2010

JEL No. I21

\section{ABSTRACT}

We motivate future neighborhood research through a simple model that considers youth educational outcomes as a function of neighborhood context, neighborhood exposure, individual vulnerability $\mathbb{R}$ neighborhood effects, and non-neighborhood educational inputs -- with a focus on effect heterogeneity. Research using this approach would require three steps. First, researchers would need to shift focus away from broad theories of neighborhood effects and examine the specific mechanisms through which the characteristics of a neighborhood might affect an individual. Second, neighborhood research would need new and far more nuanced data. Third, more research designs would be needed that can unpack the causal effects, if any, of specific neighborhood characteristics as they operate through well-specified mechanisms.

David J. Harding Department of Sociology University of Michigan Room 4128 LSA Building 500 S. State Street Ann Arbor, MI 48109-1382 dharding@umich.edu

Lisa Gennetian

The Brookings Institution 1775 Massachusetts Avenue Washington, DC 20036 gennetl@nber.org

Christopher Winship

+ DYDTF8 QYHUW

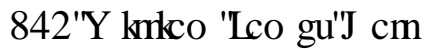

Cambridge, MA 02138

cwinship@wjh.harvard.edu
Lisa Sanbonmatsu

NBER

1050 Massachusetts Avenue, 3rd Floor

Cambridge, MA 02138

1sanbonm@nber.org

Jeffrey R. Kling

Congressional Budget Office

3403 Ordway St NW

Washington, DC 20016

and NBER

jeffrey.r.kling@gmail.com 


\section{Summary}

Most existing social science research on neighborhoods conceptualizes neighborhood effects at the macro level, hypothesizing how general neighborhood characteristics such as the poverty rate affect a variety of individual and family outcomes. Contemporary research on this topic has largely failed to recognize the diverse types of families living in poor neighborhoods or the potentially wide variety of ways that they may respond to a given set of neighborhood conditions. Our core argument is that future neighborhood research must seriously consider this diversity both conceptually and methodologically.

Our hypothesis is that there is considerable heterogeneity in the experiences of youth in the same neighborhood that might vary by their personal or family resources, their ability to cope with or navigate neighborhood circumstances, and the decisions youth and their family make about how, with whom, and where their time is spent. Depending on these and other factors, different youth may get a different "dose" of their neighborhood. This heterogeneity can generate substantial variation in how a given neighborhood characteristic affects any one youth. Youth who live in the same neighborhood may experience it in different ways, leading to "effect heterogeneity," neighborhood effects of different direction or magnitude for different youth.

With a call to pay closer attention to effect heterogeneity as the core of our argument, we motivate future neighborhood research through a simple model that considers youth educational outcomes as a function of neighborhood context, neighborhood exposure, individual vulnerability to neighborhood effects, and non-neighborhood educational inputs. Moving this research agenda forward requires three steps. First, researchers need to shift focus away from broad theories of neighborhood effects and examine the specific mechanisms through which the characteristics of a neighborhood might affect an individual. By "mechanisms" we mean the 
social, economic, and cultural processes that create associations between the compositional or demographic characteristics of neighborhoods, such as neighborhood poverty, and individual educational outcomes, such as achievement scores or educational attainment.

Second, neighborhood research desperately needs new and far more nuanced data. In particular, we need data that measure how individuals and families of different types allocate their time between different places, the extent of exposure to different people and locations, as well as the consequent influences on individual behavior.

Third, we advocate for research designs that can unpack the causal effects, if any, of specific neighborhood characteristics as they operate through well-specified mechanisms. Much current neighborhood research, in the language of structural equation models and path analysis, estimates the reduced form or total neighborhood effect. The bane of this literature has been the problem of selection —-whether differences in outcomes are due to the neighborhoods themselves or instead reflect differences in the characteristics of individuals who live in different types of neighborhoods. Rather than trying to assess the overall effect of living in a particular type of neighborhood, researchers should strive to examine discrete mechanisms in ways that account for effect heterogeneity.

Part I of this chapter introduces our conceptual framework. Part II argues for a shift from general theories to concrete specifications of mechanisms and sources of effect heterogeneity. Part III describes the need for new, detailed data on social interactions, both neighborhood and non-neighborhood based (including schools) that will allow for measurement of responses and exposure to people and places. Part IV discusses the types of research designs that might profitably be employed to estimate the effects of such interactions on educational outcomes. Part V presents a substantive example through which we illustrate one possible research design. 


\section{Introduction}

The goal of this chapter is to set the stage for future research-its opportunities as well as challenges - to better understand the influence of neighborhood social settings on youth educational outcomes. For the purposes of this chapter, what we define as a neighborhood social setting is intentionally broad: settings that are outside of home and school. We differentiate social settings according to the characteristics of the place, the types of people with whom the individual interacts, and how time is spent. Our characterization casts a wide net so that consideration may be given to a range of youth experiences, whether time spent in an afterschool program, hanging out at a basketball court or the local mall, or staying home.

Because neighborhood context is the most frequently discussed social setting, we ground our discussion in neighborhood effects on education. Our analysis is motivated by a simple yet novel conceptual framework in which a youth's educational outcome $(Y)$ is a multiplicative function of the neighborhood context $(N)$, individual exposure to that neighborhood context $(E)$, and individual vulnerability to the effects of the neighborhood context $(V)$ as well as other variables, $X . Y=\mathrm{f}(N, E, V, X)=(N \times E \times V)+X$, where each quantity potentially has multiple dimensions. The prior literature has primarily focused on estimating the effects of compositional measures of $N$ (such as neighborhood poverty rate) and on the methodological challenges of identifying the effect of $N$, particularly separating the effects of neighborhood context from the pre-existing differences between residents of different neighborhoods $(X)$.

Although selection bias remains a central issue worthy of further research, we argue that the literature has too often ignored several other key research problems that are captured in our model. The first is the mechanisms $(M)$, or social processes by which neighborhood context $(N)$ affects individual outcomes $(Y)$. The second is effect heterogeneity, or differences across 
individuals in the effects of $N$ on $Y$. This heterogeneity is driven by both $E$ and $V$. Different youth living in the same neighborhood will have different exposure $(E)$ to the people, places, and activities that drive neighborhood effects. In other words, $E$ can be thought of as the "dose" of different neighborhood characteristics that an individual receives. As Sharkey (2006) argues, individuals to some degree determine their level of exposure to different neighborhood characteristics through the decisions they (and their parents) make about where, how, and with whom to spend their time. Though the neighborhood effects literature has focused on the selection of individuals and families into neighborhoods, the selection of exposure to different neighborhood characteristics presents a second identification problem, what we might call "within-neighborhood selection bias." The social and economic processes that create differential exposure are worthy of study from both a methodological and a substantive perspective. Effect heterogeneity may also be generated by differences in the vulnerability $(V)$ or susceptibility of youth to the effects of the neighborhood $(N)$. This variation in vulnerability may be driven by differences in individual and family characteristics that make some youth more or less susceptible to neighborhood effects mechanisms. ${ }^{1}$ For example, consider the possible responses to neighborhood violence among parents of male adolescents. Some parents may require their sons to stay inside. For some this will mean more time studying; for others, more time watching TV. For the first individual, the effect of neighborhood violence will be to increase educational attainment; for the second, the effect will be neutral or to decrease educational attainment. Considerably more theorizing is needed to understand the processes behind effect heterogeneity due to both $E$ and $V$.

Although not explicitly addressed in this chapter, we think the agenda put forth here is an important step toward building evidence to inform public policy. In conceptualizing

\footnotetext{
${ }^{1} V$ may therefore be a subset of $X$.
} 
neighborhood effects, it is helpful to differentiate between interventions that are designed to affect residential mobility and interventions that are designed to directly change a place or neighborhood. In the former, an individual's neighborhood environment changes because his or her family moves to a new neighborhood, as in the Gautreaux project or Moving to Opportunity (MTO) housing mobility experiment. In the latter, the characteristics of the social setting or environment that the neighborhood provides for children is targeted for change (Sampson 2008). As we think about estimating neighborhood effects on education, we will return throughout the paper to these two types of policy interventions. In the example study design in Section V, we describe an intervention that changes individual exposure to the neighborhood.

\section{Conceptualizing Effects of Social Settings on Educational Outcomes}

In this section we motivate our critique of traditional neighborhood research by describing various mechanisms that might affect individual outcomes and in appropriate cases, why these effects might differ across families or individuals.

Environment and Health: Some of the processes through which neighborhood context may impact educational outcomes occur because of geographic location or physical proximity (rather than through local social interactions). One such mechanism operates through environmental or health effects. A neighborhood adjacent to a major highway may expose children to high levels of particulate matter pollution that leads to asthma and therefore to more school absences.

Spatial Mismatch: Another mechanism is proximity to jobs, or "spatial mismatch" (Jencks and Mayer 1990b, Mouw 2000). A neighborhood located near an abundance of job 
opportunities may affect the way that individual youth thinks about the rewards to their own education and future opportunities (Anderson 1999).

Violence: Neighborhood violence may affect the amount of time youth spend on homework if going outside means risking an experience with violence or victimization. Witnessing frequent acts of violence may lead to post-traumatic stress disorder or biological responses to stress that can also interfere with learning (Massey 2001, 2004).

Neighborhood Resources: Neighborhoods certainly differ in their institutions and other resources or resource-brokers. For example, one neighborhood may have an after-school program that provides homework help while another does not. One neighborhood may be near a community center that provides a safe, supervised space for teens to hang out, while another may not. We are only beginning to understand which resources are more or less abundant in different neighborhoods (Small 2006, 2009; Small and McDermott 2006, Small and Stark 2005). Small and McDermott (2006) find that on average poor neighborhoods actually have slightly more commercial establishments like pharmacies, grocery stores, and childcare centers, but that poor black neighborhoods with declining population density have fewer such establishments. Neighborhood resources can also fluctuate with the residential mobility of middle-class families who disproportionately sustain community institutions and organizations (Wilson 1987).

Culture: Cultural mechanisms may also be important. An example is social isolation theory (Wilson 1996, Massey and Denton 1993). According to this theory, poor inner-city black neighborhoods are thought to be socially isolated from "mainstream" or middle class individuals and institutions (particularly the labor market), leading to cultural isolation and the development of a "ghetto-specific" culture, which orients young people away from schooling by reinforcing norms and values that denigrate the value of education. A similar formulation is Fordham and 
Ogbu's (1986) oppositional culture theory. According to Fordham and Ogbu (see also Ogbu 2004), in poor black communities behaviors that promote academic achievement, such as speaking standard English, doing homework, and engaging in class discussion, become defined as "acting white" in response to discrimination, inferior schools, and blocked labor market opportunities (see also Massey and Denton 1993). However, subsequent examinations have found no evidence for the core claim that black students are disproportionately sanctioned by their peers for academic effort (Cook and Ludwig 1998, Ainsworth-Darnell and Downey 1998, Harris 2006, Carter 2005; one exception is Fryer 2006, who finds that black students who get good grades are less popular than whites who get good grades, but only in racially mixed public schools). When students' experiences with oppositional culture are investigated, we begin to see why. Carter (2005) finds that notions of "acting white" among poor black and Latino youth have more to do with musical tastes, fashion, and speech patterns than with academic performance or effort.

An alternative perspective on the cultural context of poor neighborhoods emphasizes the cultural heterogeneity of such neighborhoods. Instead of a distinct subculture, neighborhood cultural context can be conceptualized as culturally heterogeneous, incorporating competing and conflicting cultural models, some of which are shared across society more broadly and some of which are locally developed alternatives (Harding 2007, Harding 2010). For example, Carter (2005) argues that poor youth have available to them a "continuum of cultural attachments," which different youth embrace to differing degrees. For young people enmeshed in this heterogeneous cultural environment, creating and sustaining strategies for career and school success is a major challenge (Harding 2010). When alternative educational and career pathways are locally available and socially supported, some adolescents may jump to alternative pathways 
when one becomes challenging, whether or not they have full information about the risks and benefits of new strategies or the financial and cultural resources to successfully implement them.

Social Organization: Social organization theory, which focuses on the capacity of neighborhood residents to regulate behavior that occurs within the neighborhood, motivates another set of neighborhood mechanisms. Such behaviors would include schooling related behaviors of youth, such as truancy. Much of the current emphasis on the capacity of the neighborhoods focuses on behavior that occurs in public spaces, particularly crime and violence. Collective efficacy, defined as the "social cohesion among neighbors combined with their willingness to intervene on behalf of the common good," (Sampson et al.1997) mediates the relationship between compositional neighborhood characteristics (residential instability, ethnic or racial heterogeneity, and poverty) and crime rates. When parents in a community are connected to one another, what Coleman (1988) calls "intergenerational closure," they are thought to be better able to present adolescents with a consistent set of cultural ideals regarding education, leading to more school effort, and should be better able to monitor and control their children's education-related behaviors, such as attendance. However, when applied to schools, intergenerational closure has not always met with consistent empirical support (e.g., Morgan and Sorenson 1999), raising the question of whether, how, or under what circumstances socially connected parents can indeed enforce common educational ideals, and whether only proschooling cultural ideals are strengthened by intergenerational closure. It is also unclear what proportion of parents needs to be well-connected in order for the community to effectively monitor adolescent behavior. Such tipping-points or thresholds remain relatively unexamined.

Local Incentives: Finally, an economic perspective on social interactions focuses on individuals as agents responding to incentives provided by the local environment and shaped by 
their time and money resources (Manski 2000). For example, living in a neighborhood in which many students drop out of high school might reduce the stigma of dropping out and thus increase one's preference for doing so. Such a preference ordering depends on the actions of other individuals. In another neighborhood, observing neighbors who attend college and obtain a highpaying job may increase expectations about the benefits of attending college, but one unintended consequence may be a reduced number of spots in advanced placement courses. This reduces availability of such courses, but in the long-run it might also stimulate development of additional courses to meet the new demand. The difficulty is that such empirical patterns can be generated by many different interaction processes (or, even by individuals acting in isolation). In the absence of measurement of expectations and preferences, researchers are left to infer the presence of interactions from observations of outcomes.

The mechanisms we have discussed often work in tandem. For example, high collective efficacy neighborhoods may be more effective at securing outside resources, such as police protection or the resources to build and maintain a community center. Moreover, because institutions provide social contexts for the creation and maintenance of social ties, they may affect the nature and extent of neighborhood social interactions. For example, Small's research on child care centers in New York City finds, contrary to social isolation and deinstitutionalization theories, that poor neighborhoods have as many child care centers as wealthier neighborhoods, that centers in poor neighborhoods have more organizational ties to key resources, and that centers are key sites for social interaction, the creation of social ties, and resource brokering in poor neighborhoods (Small et al. 2008, Small 2009).

We note also that mechanisms often operate through parents rather than directly on children. The neighborhood environment may affect parental employment, marital decisions, 
parenting practices and psychological or physical health, and any of these can influence their children's development (for example, see Leventhal and Brooks-Gunn 2000). Parents coping with violent environments may be more likely to use physical forms of discipline because of the heightened dangers that come with misbehavior or due to the stress of living in a violent neighborhood, or parents may take cues from their neighbors when judging the academic achievement of their children. Presumably, most of any neighborhood effect on young children would operate through parenting practices or environmental mechanisms, since their direct exposure to neighborhood social interactions are likely minimal.

\section{Effect Heterogeneity}

The above theoretical perspectives provide broad outlines of how neighborhood effects on educational outcomes might work, but none of these frameworks adequately considers how these effects might differ due to differences in the daily experiences of youth. These differences, $E$, in our conceptual model in Section I, are potentially important but largely uninvestigated sources of effect heterogeneity (see also Small 2004).

One possible source of neighborhood effect heterogeneity is differences across individuals in social networks. Though interaction-based neighborhood effects theories implicitly assume that neighborhoods play some role in structuring the social networks of their residents, we actually know little about whether — or more importantly for whom — this is the case, particularly among youth. Social networks are one of the key conduits through which information and cultural frames or scripts are transmitted (but by no means the only one). Social networks of youth of a similar age ("peer networks") have received considerable attention in the literature (e.g. Anderson 1999). Such peer networks may play important roles as cultural 
conduits, as most theories of peer effects assume, but our theories need to be more specific about who those peers are, which peer attachments are more common among young people in poor neighborhoods, and what is transmitted through peer networks. Harding (2009a, 2010) argues that older adolescents and young adults on the street in poor violent neighborhoods have considerable cultural power and play an important role in socializing younger adolescents by exposing them to local cultural frames and scripts regarding schooling and sexual behavior.

A second source of effect heterogeneity is different behavioral adaptations to the challenges of daily life in poor neighborhoods. A focus on behavioral adaptations explicitly considers the individual as an actor that can adapt in different ways to mitigate or overcome challenges faced in different neighborhoods. The distinction developed by Sharkey (2006) between "imposed" environments (everything present in the neighborhood where an individual lives) and "selected" environments (the people and institutions with whom he or she interacts) highlights the idea that youth living in the same neighborhood may choose very different social environments for themselves. Different choices or adaptations can have different consequences. For example, violent neighborhoods provide particular challenges to adolescents. In order to feel safe, some adolescents may spend as little time as possible in public spaces, thus limiting their exposure to their neighborhood's violence. Others, however, may engage in behaviors such as demonstrating their toughness, forming strong bonds of mutual protection with friends, or relying on older individuals for protection in order to avoid victimization (Anderson 1999, Harding 2009a, 2010). For this later group, these same behaviors may have unintended educational consequences because they can be interpreted as resistant or disruptive by teachers (Dance 2002). Another example is provided by Carter (2005) who argues that "cultural authenticity" (in the form of speech styles, clothing, music and other tastes) among ethno-racial 
minority groups can have positive payoffs in terms of group membership and solidarity (what Carter calls "non-dominant cultural capital") but also can be misinterpreted by white middleclass teachers as oppositional or resistant. Parents may also adapt their parenting practices in response to the neighborhood environment, for example by limiting their children's interactions with neighbors (Furstenberg et al. 1999, Jarrett 1997a, b).

A third potential source of neighborhood effect heterogeneity is variation in family characteristics and the interaction between family characteristics and the properties of social settings. Here effect heterogeneity is driven less by differences in social interactions and more by differences between individuals and families in their capacity to access resources, and insulate their children from negative aspects of their neighborhood, and as a result, their susceptibility to neighborhood effects (or $V$ in our conceptual model). (Our web appendix provides a detailed example of family-based effect heterogeneity). Finally, differences across cities in economic, social, and geographic characteristics may also be an important source of heterogeneity in neighborhood effects (Small 2007).

\section{Who, When, Where and What: The Need for New Data and Methods Measuring Emergent Properties}

In order to incorporate the mechanisms and effect heterogeneity described above into neighborhood effects research, measures of exposure and vulnerability are required. With the easy availability of census data, early sociological research on neighborhood effects (e.g. Brewster 1994a,b; South and Crowder 1999, South and Baumer 2000) often relied on compositional measures of neighborhood characteristics as indicators of emergent properties (e.g. the percentage of single-mother families as a measure of cultural norms regarding non- 
marital childbearing). Economists drew liberally from these sociological roots continuing the tradition of using compositional measures. These various compositional measures (e.g. poverty rate, unemployment rate, rates of welfare receipt) tend to be fairly strongly correlated with one another. Using compositional measures of neighborhood characteristics as proxies for emergent cultural characteristics assumes a tight connection between culture and behavior, exposure, networks and interactions, when (a) this assumption is probably incorrect and (b) the connection is something we should be investigating. For example, if we observed high rates of high school dropout in poor neighborhoods, would we assume that neighborhood norms and culture did not place a high value on education? Research suggests that the poor, particularly African-American poor, actually place a very high value on education (Solarzano 1992, Goldenberg et al. 2001, Carter 2005, Young 2004, Newman 1999). More recent research has focused on developing noncompositional measures using "ecometric" methods (Sampson et al, 1997, Raudenbush and Sampson 1999). Ecometric methods are an important advance - allowing us to measure the social and cultural characteristics of neighborhoods.

Because our theories are often about emergent properties of neighborhoods rather than neighborhood composition, we need to measure emergent properties. The development of constructs and methods of data collection should be guided by relatively detailed questions such as: What are some of the dimensions that determine social interaction exposures? Where is a youth spending his or her time: inside the home, in the neighborhood, at school, or outside the neighborhood? When is the youth spending time in this environment? How long is the youth there, and where could he or she otherwise spend time? Is the youth studying, watching TV, playing sports, participating in an organized activity, or hanging out with family or friends? Who is the youth interacting with or observing: family, friends, or unrelated adults? What are the 
characteristics, experiences, attitudes, and behaviors of the people with whom the youth is interacting? What is the youth's relationship with or attitude toward these individuals (close or trusted friend, casual acquaintance, authority figure, negatively perceived)?

\section{Qualitative, Time Use and Social Network Methods}

Developing methods for measurement is as important as conceptualizing what to measure. Qualitative methods are especially well-suited to understanding social processes and day-to-day behaviors, particularly when the key dimensions the analyst might consider are not clear at the outset. By interacting with individuals in their natural social contexts or talking to them at length about their experiences and perceptions of those contexts, the ethnographer or interviewer can understand in detail how neighborhoods structure the who, when, where, and what of daily life and the content of the messages or ideas that youth encounter in these contexts.

We see three roles for qualitative methods in research on contextual effects. First, ethnographic participant observation or in-depth, unstructured interviews can be used in the pilot or exploratory phase of a project to generate hypotheses, inform the development of survey measures, or understand the boundaries of a social context in question. Second, qualitative methods can be embedded in a mixed-method study in order to understand the mechanisms by which quantitatively measured effects are operating and to inform the interpretation of estimates from statistical models. One recent example of the utility of qualitative research is the mixedmethods work in the Moving to Opportunity (MTO) evaluation. Quantitative data showed that MTO improved the outcomes of female youth, particularly their mental health, but had unfavorable effects on male youth (Kling, Liebman and Katz, 2007). The qualitative research revealed that boys in the experimental group were more socially isolated than girls in their new 
low-poverty neighborhoods, that boys in the control group experienced greater contact with father figures, and that boys generally experienced more negative peer effects. These mechanisms would have been virtually impossible to uncover with the quantitative data alone, but because qualitative data collection occurred after the quantitative work, qualitative researchers had the opportunity to explore through open-ended interviews why MTO had disparate effects. ${ }^{2}$

Third, stand-alone qualitative studies can illuminate social organization and daily life in poor communities and inform theorizing about how contextual effects operate, develop evidence for or against hypotheses based on prior research or theorizing, or complicate previous theoretical accounts. For example, Small (2004) shows that neighborhood poverty does not always lead to social disorganization and explores the conditions under which poor communities can develop and deploy social capital. A long history of ethnographic research in urban sociology has developed the "stylized facts" that now inform much of the current neighborhood effects research (e.g. Whyte 1943, Suttles 1968, Hannerz 1969, Anderson 1999, Young 2004).

Time diaries and social network analysis methods are additional tools for gathering data that can detail how, where and with whom time is spent. These methods hold considerable potential for measuring an individual's involvement in or exposure to neighborhood social processes (see, for example, Fu 2005, 2007 on network data). Traditional time use measurement studies (Juster and Thomas 1985, Robinson 1977, Csikszentmihalyi and Reed 1987, Reed 1989, and The American Time Use Survey [BLS 2009]) ask individuals to report on what they are

\footnotetext{
${ }^{2}$ In addition to the promise of MTO qualitative research for uncovering potential mechanisms, the MTO follow-up surveys collected data on items that also provide some supporting evidence for particular pathways by which MTO affected outcomes. Although the MTO study was primarily designed to answer questions about the causal effects of housing vouchers and neighborhoods and not to identify specific mechanisms, one method of gleaning possible mediating mechanisms is to examine the pattern of MTO's experimental effects on outcomes (such as math achievement) and mediators (such as school discipline) to see if the effects align with hypotheses.
} 
doing either retrospectively using a time diary or at the moment using experience sampling methods. Often data is also gathered on secondary activities (e.g. monitoring children, watching television), others present, and where the individual is (home, school, work, store, park, etc), yet when locations are requested, they are of generic form (e.g. grocery store, friend's house) and do not include geographic information that would allow researchers to measure distances or locations. We caution that time use and social network data collection can be expensive and therefore must be weighed against other data collection needs in any particular study. However, measures of social interaction in and outside of the neighborhood are critical to measuring neighborhood exposure, and data collection efforts can be efficiently tailored to these purposes. Recent work in criminology offers a promising example of how time diary methods can measure where, how, and with whom adolescents spend their time. Motivated by both a concern with neighborhoods and crime and also the routine activity theory of crime — which posits that much crime is based on spur of the moment calculations related to opportunity, potential victims or targets, and likelihood of apprehension (Cohen and Felson 1973)—criminologist Per-Olof Wikström collected time use data on a subsample of adolescents in the Peterborough Youth Study using "space-time budgets" (Wikström and Butterworth 2006). Respondents were asked to report their primary activity for each hour during the previous seven days. For each activity, they also reported where they were (including both type of location and geographic location), who else was present (e.g. number of friends, other peers, family, non-family adults, teachers, etc), whether they had consumed alcohol or drugs, whether they were engaging in crime, whether they were carrying a weapon, and whether the situation involved elements that might increase the risk of offending or victimization, such as threats, arguments, or harassment (Wikström and Butterworth 2006). Even this relatively simple form of data collection led to new descriptive 
information on adolescent criminal involvement and its relation to neighborhood context. For example, even the most frequent offenders spend very little time during the week offending; offending most often occurred with peers and in risky situations, and youth in disadvantaged neighborhoods were exposed to more risky situations. This example suggests that neighborhood effects researchers might profitably adopt and extend this form of data collection in order to examine time use and social interactions. For instance, by gathering data on the characteristics of others with whom a youth spends time, exposure to local socialization can be gauged.

\section{Measuring Institutional Resources}

"Place" can influence who individuals are interacting with, how much time they spend there (i.e. how desirable or appealing it is to engage in interactions) and can serve as a setting for the transfer of information and resources. Traditional methods to measure availability of institutions rely on geographic mapping to construct characteristics such as distance to supermarkets, or more intensive neighborhood observation check-lists that systematically ask observers to rate neighborhoods on items like presence of public playgrounds and the condition of such playgrounds (graffiti, trash, barbed wire, etc). Such methods have grown in sophistication as technologies such as "Google Earth" have vastly decreased the cost of assembling this type of information. As a result, these methods have been used to address a variety of public health questions about the role of social and physical environments in health outcomes among individuals living in disadvantaged communities (e.g. see Zenk et al 2005, Ponce et al 2005).

While mapping or collecting rater-observations (also called "Systematic Social Observation") is a good strategy for documenting the number and proximity of these types of 
neighborhood institutions, these methods, as argued by Sampson, Morenoff and Gannon-Rowley (2002), do not capture the quality and diversity of available institutions, nor the commonality and acceptance of their use by residents (gathering places, trusted pharmacies, or safe parks). Recent work in public health examining supermarket availability in impoverished neighborhoods concludes, for example, that travel time may be a better indicator of accessibility than physical distance (Zenk et al 2005). A fuller understanding of institutional resources can complement how well researchers understand where and how time is spent and how to characterize place. Retrieving this type of data may require a hybrid model that combines low-cost methods (mapping and counting) with more resource intensive but tailored methods such as neighborhood observation check-lists along with individual qualitative assessments of institutions (via a subsample of survey respondents or respondents to more in-depth open-ended interviews).

\section{Estimation Strategies: Effects of Causes, Effect heterogeneity, and Mechanisms Selection Bias}

"Selection bias" problems present themselves when families and individuals have some control over where they live, with whom they interact, and where they spend their time. Individuals make decisions about the social settings they occupy based on a variety of factors, from preferences to personal resources and other constraints, and because researchers cannot always observe or measure these factors, selection bias may result. This means that individual or family characteristics may confound the estimates of social setting on youth outcomes because the associations we observe between a social setting such as neighborhood context and educational outcomes may be due to unobserved differences in individual or family characteristics across neighborhoods and not to the effects of residing in different 
neighborhoods. The magnitude of the bias will depend on two quantities: the association between the confounder and social setting, and the association between the confounder and the outcome.

A useful way of conceptualizing this identification problem is to consider the sources of variation in social settings experienced by different youth. Take, for example, neighborhood context. One type of variation, endogenous variation, refers to the confounders that produce selection bias discussed above. The second type of variation is exogenous variation in neighborhood context, variation that is produced by economic or social processes that do not directly affect individual outcomes. For example, a change in public policy may move some families out of public housing into neighborhoods with lower poverty rates without directly affecting outcomes of interest.

Solving the selection bias problem requires finding and measuring those exogenous sources of variation. In searching for such exogenous variation in neighborhood contexts, researchers have focused on two types of processes that lead to variation in neighborhoods across individuals: (1) residential mobility, or the movement of families from one neighborhood to another, and (2) changes in neighborhood conditions over time, i.e. a "place-based" change. Since both sources of variation can be endogenous or exogenous, both present identification challenges. In the former, we must understand why some families move to (or stay in) disadvantaged neighborhoods while others do not. Moreover, we must consider the potential negative effects of residential mobility itself, which could dilute the positive effects of an improved neighborhood context (Sampson 2008). ${ }^{3}$ In the latter, we must understand why some neighborhoods change and some do not, and we may also worry about why some families move

\footnotetext{
${ }^{3}$ Sobel (2006) discusses another identification problem in mobility experiments, interference between units that we do not fully describe here. This is a particular challenge in this context because encouraging and succeeding in changing residential mobility inherently can have possible confounding effects on the families in the receiving neighborhood as well as those in the neighborhood left behind.
} 
in response to changes in their neighborhoods and others do not. If these reasons are also causally related to the outcomes, selection bias is introduced. Finally, note that these two types of interventions typically will not estimate the same quantity. Residential mobility manipulates an entire set of linked neighborhood characteristics, while a place-based intervention typically

manipulates a single key feature (or small number of key features) of the neighborhood context. A place-based intervention will therefore more often provide a narrow test of a specific neighborhood effects mechanism. For this reason, and because we have already learned much from residential mobility studies like MTO and Gautreaux, we support the development of placebased interventions for studying neighborhood effects. Key challenges in developing such interventions include designing interventions that are strong enough to produce detectable effects and specific enough to reveal the importance of a single mechanism.

\section{Identifying the effects of specific mechanisms}

Focusing on mechanisms introduces an additional set of complications to research designs that rely on harnessing exogenous variation in social settings. Though not cast precisely in terms of mechanisms, it is helpful to start with Manski's discussion of identification problems in neighborhood effects research since his framework is well-known and the fundamental problems of interpreting associations between contextual characteristics and individual outcomes are especially relevant in identifying mechanisms (Manski 1993, 1995). Manski describes three sources of association between neighborhood characteristics and individual outcomes. First, "endogenous effects" are the effects of group level values on individual values on the same variable. For example, contagion or peer effects theories propose that individuals are more likely to do what others around them are doing. A child will spend more time studying when he sees 
his peers spending more time studying. Endogenous effects generate a social multiplier because they amplify any direct effect of an intervention. These types of effects are particularly challenging to identify due to direction of causality, or what Manski calls the "reflection problem." Is directionality from the group to the child or vice versa? Second, in "contextual effects," individual behavior varies with other characteristics of the group, such as achievement varying with neighborhood socio-economic composition. A child may spend more time studying when he observes adults in the neighborhood who have benefitted from high levels of education. Third are "correlated effects," which is simply another name for selection bias.

If we want to identify the total effect of exposure to one neighborhood rather than another, then it is not necessary to distinguish between endogenous and contextual effects with respect to the mechanisms that they specify. The main concern is selection bias at the neighborhood level, as discussed above. (Note, however, that even if we were able to deal with the selection bias problem and obtain a causal estimate of the effect of, say, neighborhood poverty on an educational outcome, this would not distinguish between the effects of neighborhood poverty and other neighborhood characteristics correlated with it.)

The reflection problem emerges whenever we are interested in endogenous effects of social settings, but it is not the same identification problem as the selection bias problem researchers face when trying to estimate total effects. The reflection problem can be understood as a failure to specify, measure, and manipulate (or find an instrument for) one particular mechanism, the average value of the outcome among a group with whom one interacts. The only other option is to draw on theory to invoke strong identifying assumptions that specify the direction of causality. For instance, perhaps older friends' actions affect younger friends' actions, but not the reverse. The key point is that the reflection problem is not an inherent intractable 
problem in the estimation of neighborhood effects but rather results from failure to conceptualize mechanisms and develop strategies for identifying their effects.

We now return to the selection bias problem, but consider it in the context of identifying the role of mechanisms. Even if there is a source of random variation in neighborhood context, the selection bias problem re-emerges when mechanism variables are considered, as selfselection into the mechanisms may no longer be random with respect to the outcomes. Identifying the effects of mechanisms on an outcome will require multiple sources of exogenous variation. Consider Figure 1, which diagrams a simplified research design in which there are three hypothesized mechanisms (M1, M2, M3) for the effect of N (a neighborhood characteristic) on $\mathrm{Y}$ (the outcome). For example, $\mathrm{N}$ might be the amount of violence in the neighborhood and Y might be educational achievement. The three mechanisms might be (M1) leveling of educational expectations through a focus on safety, (M2) exposure to violence affecting cognitive development through Post Traumatic Stress Disorder, and (M3) joining a gang for protection leading to less time for studying. ${ }^{4} \mathrm{U}$ represents a set of unobserved $\mathrm{X}$ variables that are uncorrelated with Z (since it was randomly assigned) but are correlated with neighborhood violence $(\mathrm{N})$, the outcome $(\mathrm{Y})$, and the mechanisms $\left(\mathrm{M}\right.$ 's). ${ }^{5}$

\section{-- FIGURE 1 HERE --}

If we are interested in the total effect of neighborhood violence $(\mathrm{N})$ on educational achievement (Y), we can use instrumental variables to estimate the effect. This approach involves finding another variable, $\mathrm{Z}$, that is correlated with neighborhood violence $(\mathrm{N})$ and uncorrelated with the unobserved variables $(\mathrm{U})$. $\mathrm{Z}$ is the source of exogenous variation in our

\footnotetext{
${ }^{4}$ Note that one might further theorize mechanisms for these mechanisms. The level of detail in the specification of mechanisms depends on one's substantive and policy goals. See Morgan and Winship (2007, Ch. 8) for discussion of this issue.

${ }^{5}$ For simplicity we omit the observed X's from the figure. The discussion in this section will assume conditioning on observed $\mathrm{X}$ variables.
} 
neighborhood characteristic (N). For example, the city might randomly assign an anti-violence program to some neighborhoods and not others. Referred to as an instrument, $\mathrm{Z}$ is used to purge $\mathrm{N}$ of the portion of its variation that is correlated with the unobservables. The exogenous portion of N's variation - that is, the uncorrelated portion that remains — is then used to estimate the effect of $\mathrm{N}$ on $\mathrm{Y}$. Intuitively, this means that our analysis is using only the variation in neighborhood violence created by the anti-violence program to identify the effects of violence. Because the anti-violence program is randomly assigned, the communities that did not get the anti-violence program are similar in observed and unobserved ways to the communities that did. We can use a similar procedure to identify the effect of the neighborhood characteristic $(\mathrm{N})$ on each of the mechanisms. For example, b1 can be estimated as the association between the anti-violence program (Z) and educational expectations (M1) divided by the effect of the program on neighborhood violence (a). The difficulty is that as long as each of the mechanisms (M's) is associated with the outcome Y through the unobserved variables (U), we have no way to identify the effects of these mechanisms on educational achievement without more instruments, i.e. without sources of exogenous variation in the mechanisms. Moreover, if the mechanisms are causally related to one another, then even if we could measure all the U's, we would also have to assume that we have measured all the mechanisms as well, lest our estimate of the effect of any one mechanism on $\mathrm{Y}$ be biased by its association with an unobserved mechanism. If we thought that violence also directly reduced school attendance and that educational expectations are correlated with school attendance but we did not observe school attendance, we might attribute some of the effects that operate solely through school attendance to educational expectations. One way to create the additional exogenous variation necessary to identify the effects of specific 
mechanisms (c's) is to design an experiment with multiple treatment arms that produce exogenous random variation in both $\mathrm{N}$ and the M's across the treatment arms.

Another general approach in an experimental setting is to examine patterns of effects on mediating mechanisms by subgroups. This can be useful when the experiment is not designed with multiple treatment arms for the various mechanisms, and can be done by dividing the sample into subgroups for which theory and previous evidence suggest that treatment effects of $\mathrm{N}$ on $\mathrm{Y}$ may differ. A review of the overall pattern of results can provide some evidence to support a theoretical framework by testing, in part, the hypothesized relationships between mechanisms and outcomes across treatment categories and subgroups. The advantage of this type of experimental approach is that the source of variation in the social setting (e.g. from something like randomly assigned rent subsidies that affect residential location) is clear, so we can be confident that changes in the mechanisms are causally related to residential location. However, without an exogenous source of variation in the mechanisms, the mechanisms may be spuriously correlated with the outcomes through unobservables.

Focusing on mechanisms can also strengthen our confidence in causal claims. Consider again Figure 1 in which there are three mechanisms (M's) and some set of unobserved confounders (U). If any one of the observed mechanisms is uncorrelated with $U$, then we can identify the portion of the effect of the treatment $(\mathrm{N})$ on the outcome $(\mathrm{Y})$ that operates through that mechanism. For example, if we can assume that the "joining gangs" mechanism (M3) is not affected by unobservables (U), then the arrow between $U$ and M3 disappears from the diagram. When M3 is not affected by $\mathrm{U}$, and when we can observe the other mechanisms, both the effect (b3) of the treatment on joining gangs and the effect (c3) of joining gangs on achievement can now be identified even if there is no instrument. The effect of the neighborhood violence (N) on 
achievement (Y) that operates through joining gangs (M3) can be calculated as the product of b3 and c3. While this does not provide us with an unbiased estimate of the total effect of $\mathrm{N}$ on $\mathrm{Y}$, if $\mathrm{b}^{*}{ }^{*} \mathrm{c} 3$ is nonzero, it does provide evidence that there is some nonzero effect of $\mathrm{N}$ on $\mathrm{Y} .{ }^{6}$

\section{Complications introduced by effect heterogeneity}

In the above example the subgroups are observed and effect heterogeneity is harnessed to provide further information. The possibility of heterogeneity in effects also introduces complications, particularly when the subgroups cannot be identified in the data. The first complication has already been discussed in the methodological literature on instrumental variables estimation, that is, unless the treatment effect can be assumed to be the same for all units of analysis, an instrument identifies the local average treatment effect (LATE) (Angrist, Imbens and Rubin, 1996; Angrist and Krueger, 2001; and Gennetian et al., 2005, for a less technical exposition). It answers the question: What is the average effect of the treatment among those who were actually induced to take the treatment by the instrument? The conventional solution to the LATE problem is to use multiple instruments (see applications by Kling, Liebman and Katz, 2007; Gennetian, Magnuson and Morris, 2008). If different LATE estimates from different instruments operate in the same direction and are of similar magnitude, then we can be more confident that the effect of the treatment is not specific to particular subgroups.

A second complication arises because estimating the role of a particular mechanism in the effect of a neighborhood characteristic $(\mathrm{N})$ on an educational outcome $(\mathrm{Y})$ requires identifying both the effect of $\mathrm{X}$ on the mechanism and the effect of the mechanism on $\mathrm{Y}$. When there is treatment effect heterogeneity, it is possible to produce an estimate of the role of the

\footnotetext{
${ }^{6}$ This discussion is an example of identification through what Pearl (2000) calls his "front door" criterion. See Winship and Harding (2008) for an example and further details.
} 
mechanism that applies to none of the cases if some cases experience effects of $\mathrm{N}$ on the mechanism and other cases experience an effect of the mechanism on $\mathrm{Y}$ (see also Bullock and Ha 2010). In technical terms, this means that the LATE for the effect of $\mathrm{N}$ has a different group of compliers than the LATE for the effect of M.

This problem highlights the importance of specifying and measuring sources of effect heterogeneity. It is particularly important to choose exogenous family or child characteristics, lest subgroup membership be a product of the treatment. Ideally one might also purposely stratify one's sample to ensure sufficient sample size in each subgroup. Our discussion thus far suggests a number of other family or child characteristics on which one might focus. Our emphasis on family capacity or family resources suggests that family income, family structure, or number of children, or access to transportation may determine the strength of a neighborhood effect. Our emphasis on social ties and interactions in the neighborhood suggests that proximity to extended kin, parents' social ties to the neighborhood, or parental employment outside the neighborhood may determine the strength of the neighborhood effect. Many of these characteristics are both determinants and effects of the treatment. Further ethnographic work may be required to better understand these and other sources of effect heterogeneity. ${ }^{7}$ We emphasize as well that an exhaustive search for effect heterogeneity may lead to multiple testing problems through the mining of sample data for statistical significance. Sources of effect heterogeneity should be closely informed by theory and prior research and any analyses should be preceded by a clear set of hypotheses limiting the number of subgroups to be examined.

\section{An Illustrative Example}

\footnotetext{
${ }^{7}$ One might also consider family type to be a latent class and conduct a latent class analysis.
} 
Because violence is spatially concentrated in poor neighborhoods, violence may be an important mechanism linking neighborhood poverty to school outcomes. In this section we sketch an example study that examines whether the observed correlation between neighborhood violence and unfavorable youth schooling outcomes (e.g. Harding 2009b) is causal by manipulating exposure to neighborhood violence through youth time use. Suppose we observe that youth living in more violent neighborhoods have lower school attendance and graduation rates, high rates of suspensions, and poorer math achievement. One can try to test the neighborhood violence hypothesis by estimating the relationship between an individual's time spent on the streets in a violent neighborhood and educational achievement using a regression model, but this estimate will be susceptible to within-neighborhood selection bias (different youth choosing to spend their time in different places, in different ways, and with different people). An alternative is to identify this effect by generating exogenous variation in youth time use through random assignment.

We can manipulate the amount of time youth spend in areas of higher neighborhood violence under the hypothesis that such time use affects exposure to violence. Randomization ensures that individuals assigned to different locations are the same across both observed and unobserved characteristics, except for chance variation, so any differences in outcomes across groups can be attributed to the planned variation in exposure to violence.

Details of this example study can be found in the web appendix. Briefly, the design is the following. In the first stage, we select a target set of neighborhoods with high neighborhood violence and match each of them to neighborhoods that are comparable in terms of poverty, race, and educational levels but have lower neighborhood violence (see Seith et al, 2003 on the feasibility of matched-neighborhood designs). An intervention would be designed that is non- 
academic, but involves engagement with the neighborhood - such as working on a local clothing drive. For the study, we would recruit individuals from the neighborhoods with higher levels of neighborhood violence, so that the neighborhoods where subjects are placed through the intervention are all less risky than their own neighborhoods. We would randomly assign these youth to teams in different locations outside of their own neighborhoods, some of which would have lower neighborhood violence than others.

With this type of random assignment study design we can learn about the effect of exposure to neighborhood violence by comparing the educational outcomes of youth in the different intervention groups. Although we do not directly manipulate exposure to violence, we can estimate the intervention's impact on educational outcomes and the intervention's impact on exposure to violence. For example, we hypothesize that youth working in less violent neighborhoods would experience more beneficial educational effects than those working in more violent neighborhoods. The benefits of the interventions may vary at the individual level by preintervention exposure and vulnerability to neighborhood violence, as determined by family connections to resources outside of the neighborhood, parenting skills, parental motivation and capacity to seek positive alternative environments for children, and the youth's own social relationships and decisions about how to use their time.

Measures of youth time use, neighborhood characteristics and exposure to violence, and a range of information about families and their youth can be collected through surveys. With these data, we can also check for any other important differences in youth experiences that arise during the study which may be correlated with exposure to violence. Survey data can be complemented by in-depth qualitative interviews with a small subsample of youth in both the experimental and 
control groups to better understand aspects of youth time use and exposure to violence which are difficult to detect or measure through a survey.

\section{Conclusion}

Our aim in this chapter has been to set the stage for future research-its opportunities as well as challenges, both conceptually and empirically — to better understand the influence of neighborhood social settings on youth educational outcomes. Conceptually, we argue that the field is ready to move away from estimating the effects of compositional properties of neighborhoods and toward an analysis of specific mechanisms and effect heterogeneity. A new focus on mechanisms, exposure, and vulnerability can only be achieved with more nuanced data. Our recommendation is to build on the successes of prior efforts and invest in new, mixed methods data collection strategies that can measure individual interactions, networks, and time use and provide reliable assessments of neighborhood resources, cultural contexts, and physical conditions. We see particular promise in adapting time use and social network data collection methods to assess exposure to the social and cultural processes that produce contextual effects. Finally we point to both across neighborhood and within neighborhood selection biases as longstanding challenges in identifying neighborhood effects and illustrate some promising approaches for designing studies to identify mechanisms and assess effect heterogeneity. Although we tailor our discussion to social settings outside of the school and home, much of what we discuss might also be applied to school settings or home environments.

Neighborhoods are not static, nor are individual residential decisions. Our proposed framework recognizes this to some extent by highlighting the importance of effect heterogeneity. We have not addressed other forms of neighborhood dynamics. Neighborhoods change over time 
as residents move in and out, and community investment increases or decreases. When families move in response to changes in the neighborhoods around them or in response to the outcomes experienced by their children as a result of neighborhood context, then more sophisticated statistical methods, such as marginal structural models or inverse probability of treatment weighting (Robins, Hernan, and Brumback 2000), are required to estimate unbiased causal effects, even when all sources of selection bias are accurately measured (Sampson, Sharkey and Raudenbush 2008, see also Sharkey and Sampson forthcoming).

We have discussed identification of mechanisms for neighborhood effects without regard to the absolute magnitude of these effects, to their relative magnitudes compared to the effects of schools or of families, or to their resulting policy significance. Policy trade-offs between housing vouchers that offer the opportunity to move compared to place-based neighborhood investment (such as building a community center or clinic) are best informed by quantifying the size of effects and comparing costs and benefits. Mechanisms by which neighborhoods can have their effects are particularly important in considering these types of policy trade-offs. For example, if mix of peers in neighborhood-based after-school activities is an important predictor of youth math achievement, then policy makers can evaluate the size of this beneficial peer effect (and any spillover effects) compared to the cost of busing disadvantaged youth to programs that offer this same mix of peers, or the cost of offering an equivalent program in the schools attended by disadvantaged youth. Heterogeneous treatment effects are equally important in considering policy trade-offs. Policy interventions that primarily benefit only some subgroups may be more or less appealing. For example, an intervention with a large effect on a small group of youth may be more or less appealing than an intervention with a small effect on a very large group of youth. 


\section{References}

Ainsworth-Darnell, James and Douglas Downey. 1998. "Assessing the Oppositional Culture Explanation for Racial/Ethnic Differences in School Performance." American Sociological Review 63:536-553.

Anderson, Elijah. 1999. Code of the Street: Decency, Violence, and the Moral Life of the Innercity. New York: W. W. Norton.

Angrist, Joshua and Alan Krueger 2001. "Instrumental Variables and the Search for Identification: From Supply and Demand to Natural Experiments." Journal of Economic Perspectives, 15(4), 69-85.

Angrist, Joshua, Guido Imbens, and Donald Rubin 1996. "Identification of Causal Effects Using Instrumental Variables." JASA Applications Invited paper, with comments and authors' response. Journal of the American Statistical Association, 91, 444-455.

Brewster, Karin L. 1994a. "Race Differences in Sexual Activity Among Adolescent Women: The Role of Neighborhood Characteristics." American Sociological Review 59: 408-424.

Brewster, Karin L. 1994b. "Neighborhood Context and the Transition to Sexual Activity among Young Black Women." Demography 31: 603-614.

Brooks-Gunn, Jeanne, Greg J. Duncan, Pamela K. Klebanov, and Naomi Sealand. 1993. "Do Neighborhoods Influence Child and Adolescent Development?" American Journal of Sociology 99:353-95.

Browning, Christopher R., Tama Leventhal, and Jeanne Brooks-Gunn. 2004. "Neighborhood Context and Racial Differences in Early Adolescent Sexual Activity." Demography 41: 697-720.

Browning, Christopher R., Tama Leventhal, and Jeanne Brooks-Gunn. 2005. "Sexual Initiation in Early Adolescence: The Nexus of Parental and Community Control." American Sociological Review 70: 758-778.

Bullock, John G., and Shang E. Ha. 2010. "Mediation Analysis Is Harder than It Looks.” In Cambridge Handbook of Experimental Political Science, ed. James N. Druckman, Donald P. Green, James H. Kuklinski, and Arthur Lupia. New York: Cambridge University Press.

Bureau of Labor Statistics. 2009. American Time Use Survey User's Guide. http://www.bls.gov/tus/atususersguide.pdf (accessed February 25, 2010).

Carter, Prudence L. 2005. Keepin' It Real: School Success Beyond Black and White. New York: Oxford University Press. 
Cohen, Lawrence E., and Marcus Felson. 1979. "Social Change and Crime Rate Trends: A Routine Activity Approach." American Sociological Review 44 (4): 588-608.

Coleman, James S. 1988. "Social Capital in the Creation of Human Capital." American Journal of Sociology 94: S95-S120

Cook, Phillip and Jens Ludwig. 1998. "The Burden of 'Acting White': Do Black Adolescents Disparage Academic Achievement?” Pp. 375-401 in Christopher Jencks and Meredith Phillips (eds) The Black White Test Score Gap. Washington, D.C.: Brookings.

Cook, Tom D., William J. Shadish, and Victor C. Wong. 2008. "Three Conditions Under Which Observational Studies Produce the Same Results as Experiments.” Journal of Policy Analysis and Management 27(4): 724-750.

Csikszentmihalyi, Mihaly and Larson, Reed. 1987. "Validity and Reliability of the Experience Sampling Method," Journal of Nervous and Mental Disease, 175: 526-536.

Currie, Janet, Eric Hanushek, E. Megan Kahn, Matthew Neidell and Steve Ridkin. 2007. "Does Pollution Increase School Absences?” National Bureau of Economic Research Working Paper No. 13252.

Dance, L. Janelle. 2002. Tough Fronts: The Impact of Street Culture on Schooling. New York: Routledge Falmer Press.

Fordham, Signithia and John Ogbu. 1986. "Black Students' School Success: Coping with the Burden of "Acting White." Urban Review 18(3):176-206.

Fryer, Roland G. 2006. "Acting White: The Social Price Paid by the Best and brightest Minority Students.” Education Next (Winter 2006): 53-59.

Fu, Yang-chih. 2005. "Measuring personal networks with daily contacts: a single-item survey question and the contact diary." Social Networks 27: 169-86.

Fu, Yang-chih. 2007. "Contact diaries: Building archives of actual and comprehensive personal networks.” Field Methods 19: 194-217

Furstenburg, Frank F. et al (eds). 1999. Managing to Make It: Urban Families and Adolescent Success. Chicago: University of Chicago Press.

Gennetian, Lisa, Lisa Sanbonmatsu and Jens Ludwig. 2009. "Neighborhood Poverty Effects on Low Income Youth: A Within Study Comparison Using Data from the Moving to Opportunity Study." Working Paper. Presented at 2009 Society For Research in Child Development, Denver, Colorado.

Gennetian, Lisa, Katherine Magnuson and Pamela Morris. 2008. "Statistical Association to Causation: What Developmentalists Can Learn from Instrumental Variables Techniques 
Coupled with Experimental Data." Developmental Psychology. 44(2): 381-394.

Gennetian, Lisa., Pamela Morris, Johannes Bos and Howard Bloom. 2005. "Coupling the Nonexperimental Technique of Instrumental Variables with Experimental Data to Learn How Programs Create Impacts." Chapter 3 in H. Bloom (ed.) Moving to the Next Level: Combining Experimental and Non-Experimental Methods to Advance Employment Policy Research. New York, NY: Russell Sage Foundation.

Goldenberg, Claude, Ronald Gallimore, Leslie Reese, and Helen Garnier. 2001. "Cause or Effect? A Longitudinal Study of Immigrant Latino parents' Aspirations and Expectations, and Their Children's School Performance." American Educational Research Journal 38(3): 547-582.

Grannis, Rick. 1998. "The Importance of Trivial Streets: Residential Streets and Residential Segregation." American Journal of Sociology 103(6): 1530-64.

Hannerz, Ulf. 1969. Soulside: Inquiries into Ghetto Culture and Community. New York: Columbia University Press.

Harding, David J. 2007. "Cultural Context, Sexual Behavior, and Romantic Relationships in Disadvantaged Neighborhoods." American Sociological Review 72(3): 341-364.

Harding, David J. 2009a. "Violence, Older Peers, and the Socialization of Adolescent Boys in Disadvantaged Neighborhoods." American Sociological Review 74(3): 445-464.

Harding, David J. 2009b. "Collateral Consequences of Violence in Disadvantaged Neighborhoods.” Social Forces 88(2): 757-782.

Harding, David J. 2010. Living the Drama: Community, Conflict, and Culture Among Inner-City Boys. Chicago: University of Chicago Press.

Harris, Angel L. 2006. “I (Don’t) Hate School: Revisiting Oppositional Culture Theory of Blacks' Resistance to Schooling.” Social Forces 85: 797-834.

Hollister, Robinson and Elizabeth Ty Wilde. 2007. "How Close is Close Enough? Evaluating Propensity Score Matching Using Data from a Class Size Reduction Experiment." Journal of Policy Analysis and Management 26(3): 455-477.

Hoxby, C. M. 2001. “All School Finance Equalizations Are Not Created Equal.” The Quarterly Journal of Economics, 116, 1189-1230.

Jacob, Brian 2004. "Public Housing, Housing Vouchers and Student Achievement: Evidence from Public Housing Demolitions in Chicago." American Economic Review. 94(1): 233258. 
Jarrett, Robin L. 1997a. “African American Family and Parenting Strategies in Impoverished Neighborhoods," Qualitative Sociology 20: 275-288.

Jarrett, Robin L. 1997b. "Bringing Families Back In: Neighborhood Effects on Child Development.” Pp. 48-64 in Jeanne Brooks-Gunn, Greg J. Duncan, and J. Lawrence Aber, eds. Neighborhood Poverty, Vol. 2: Policy Implications in Studying Neighborhoods. New York: Russell Sage

Jencks, Chrsitopher and Susan E. Mayer. 1990a. "The Social Consequences of Growing Up in a Poor Neighborhood," p. 111-186 in Lawrence E. Lynn, Jr. and Michael G. H. McGreary, eds. Inner-City Poverty in the United States. Washington, D.C.: National Academy Press.

Jencks, Chrsitopher and Susan E. Mayer. 1990b. "Residential Segregation, Job Proximity, and Black Job Opportunities: The Empirical Status of the Spatial Mismatch Hypothesis" in Inner-City Poverty in the United States edited by Lawrence Lynn, Jr. and Michael McGeary. Washington, D.C.: National Academy of Sciences Press.

Juster, F. Thomas and Stafford, Frank P. (eds.) 1985. Time, Goods, and Well-Being. Ann Arbor, Michigan: Institute for Social Research, University of Michigan.

Kling, Jeffrey R., Jeffrey B. Liebman, and Lawrence Katz. 2007. "Experimental Analysis of Neighborhood Effects." Econometrica 75(1): 83-119.

Krieger N, Chen JT, Waterman PD, Soobader MJ, Subramanian SV, Carson R. 2002. "Geocoding and Monitoring of US Socioeconomic Inequalities in Mortality and Cancer Incidence: Does the Choice of Area-Based Measure and Geographic Level Matter?: the Public Health Disparities Geocoding Project.” American Journal of Epidemiology. 156:471-482.

LaLonde, Robert J. 1986. "Evaluating the Econometric Evaluations of Training Programs with Experimental Data.” American Economic Review 76:604-20.

Larson, Reed. 1989. "Beeping Children and Adolescents: A Method for Studying Time Use and Daily Experience," Journal of Youth and Adolescence, 18: 511-530.

Leventhal, Tama and Jeanne Brooks-Gunn 2000. "The Neighborhoods they Live In: The Effects of Neighborhood Residence Upon Child and Adolescent Outcomes.” Psychological Bulletin, 126, 309-337.

Manski, Charles F, 1993. "Identification of Endogenous Social Effects: The Reflection Problem," Review of Economic Studies 60(3):531-42.

Manski, Charles F. 1995. Identification Problems in the Social Sciences. Cambridge, MA: Harvard University Press. 
Manski, Charles. 2000. Economic Analysis of Social Interactions. Journal of Economic Perspectives (14)3: 115-136.

Massey, Douglas S. 2001. "Segregation and Violent Crime in Urban America." Pp. 317-345 in Elijah Anderson and Douglas S. Massey, eds. Problem of the Century: Racial Stratification in the United States. New York: Russell Sage.

Massey, Douglas S.. 2004. "Segregation and Stratification: A Biosocial Perspective," Du Bois Review 1: 7-25.

Massey, Douglas S. and Nancy Denton. 1993. American Apartheid: Segregation and the Making of an Underclass. Cambridge, MA: Harvard University Press.

Michalopoulos, Charles, Howard S. Bloom and Carolyn J. Hill. 2004. "Can Propensity-Score Methods Match the Findings from a Random-Assignment Evaluation of Mandatory Welfare-to-Work Programs?" Review of Economics and Statistics. 86(1): 156-179.

Moffitt, Robert. 2004. "Policy Interventions, Low-Level Equilibria, and Social Interactions." Pgs 45-82 in Steven N. Durlauf and H. Peyton Young (eds) Social Dynamics. Washington, DC: Brookings Institution Press.

Morgan, Stephen L. and Aage B. Sørensen. 1999. "Parental Networks, Social Closure, and Mathematics Learning: A Test of Coleman's Social Capital Explanation of School Effects." American Sociological Review 64:661-681.

Morgan, Stephen L. and Christopher Winship. 2007. Counterfactuals and Causal Inference: Methods and Principles for Social Research. Cambridge: Cambridge University Press.

Mouw, T. 2000. "Job Relocation and the Racial Gap in Unemployment in Detroit and Chicago, 1980 to 1990." American Sociological Review 65 5:730-753.

Newman, Katherine S. 1999. No Shame in My Game: The Working Poor in the Inner-city. New York: Vintage and Russel Sage.

Ogbu, John U. 2004. "Collective Identity and the Burden of 'Acting White' in Black History. Community, and Education." The Urban Review 36: 1-35.

Pearl, Judea. 2000. Causality: Models, Reasoning, and Inference. Cambridge, UK: Cambridge University Press.

Ponce, Ninez A. Katherine J. Hoggatt, Michelle Wilhelm, and Beate Ritz. Preterm Birth: The Interaction of Traffic-related Air Pollution with Economic Hardship in Los Angeles Neighborhoods American Journal of Epidemiology, 162(2): 140-148. 
Raudenbush, Stephen W, and Sampson, Robert J. 1999. "Ecometrics: Toward a Science of Assessing Ecological Settings, with Application to the Systematic Social Observation of Neighborhoods." Sociological Methodology 29: 1-41.

Robins, James A., Miguel A. Hernan, and Babette Brumback. 2000. "Marginal structural models and causal inference in epidemiology." Epidemiology 11 5:550-560.

Robinson, John P. 1977. How Americans Used Time in 1965. Ann Arbor, Michigan: Institute for Social Research.

Sampson, Robert, Stephen Raudenbush, and Felton Earls. 1997. "Neighborhoods and Violent Crime: A Multilevel Study of Collective Efficacy." Science. 227:918-924.

Sampson, Robert J., Jeffrey D. Morenoff and Thomas Gannon-Rowley. 2002. “Assessing Neighborhood Effects: Social Processes and New Directions in Research.” Annual Review of Sociology 28:443-478.

Sampson, Robert J. and Steve Raudenbush. 1999. "Systematic Social Observation of Public Spaces: A New Look at Disorder in Urban Neighborhoods." American Journal of Sociology 105: 603-651.

Sampson, Robert J., Patrick Sharkey, and Stephen Raudenbush. 2008. "Durable Effects of Concentrated Disadvantage on Verbal Ability among African-American Children." Proceedings of the National Academy of Sciences 105:845-852.

Sampson, Robert J. 2008. "Moving to Inequality: Neighborhood Effects and Experiments Meet Social Structure" American Journal of Sociology 114: 189-231.

Sharkey, Patrick T. 2006. "Navigating Dangerous Streets: The Sources and Consequences of Street Efficacy." American Sociological Review 71: 826-846.

Sharkey, Patrick T. and Robert J. Sampson. Forthcoming. "Destination Effects: Residential Mobility and Trajectories of Adolescent Violence in a Stratified Metropolis." Criminology.

Sieth, David C., Verma, Nandita, Bloom, Howard S. and George C. Galster. 2003. "Exploring the Feasibility and Quality of Matched Neighborhood Research Designs.” MDRC. Working Papers on Research Methodology.

Solorzano, Daniel G. 1992. "An Exploratory Analysis of the Effects of Race, Class and Gender on Student and Parent Mobility Aspirations." Journal of Negro Education 61: 30-44.

Small, Mario L. 2004. Villa Victoria: The Transformation of Social Capital in a Boston Barrio. Chicago: University of Chicago Press. 
Small, Mario L. 2006. "Neighborhood Institutions as Resource Brokers: Childcare Centers, Inter-Organizational Ties, and Resource Access among the Poor." Social Problems 53:274-92.

Small, Mario L. 2007. "Is There Such a Thing as 'The Ghetto'? The Perils of Assuming that the South Side of Chicago Represents Poor Black Neighborhoods.” City 11(3):413-21.

Small, Mario L. 2009. Unanticipated Gains: Origins of Network Inequality in Everyday Life. New York: Oxford University Press.

Small, Mario Luis, Erin M. Jacobs, and Rebekah P. Massengill. 2008. "Why Organizational Ties Matter for Neighborhood Effects: A Study of Resource Access through Childcare Centers." Social Forces 87(1).

Small, Mario L. and Monica McDermott. 2006. "The Presence of Organizational Resources in Poor Urban Neighborhoods: An Analysis of Average and Contextual Effects." Social Forces. 84: 1697-1724.

Small, Mario L. and Laura Stark. 2005. "Are Poor Neighborhoods Resource-Deprived? A Case Study of Childcare Centers in New York.” Social Science Quarterly 86:1013-36.

Stack, Carol. 1974. All Our Kin. New York: Basic Books.

Sobel, Michael. 2006. "What Do Randomized Studies of Housing Mobility Demonstrate? Causal Inference in the Face of Interference." Journal of the American Statistical Association 101 (476): 1398-1407.

South, Scott J. and Kyle D. Crowder. 1999. "Neighborhood Effects on Family Formation: Concentrated Poverty and Beyond." American Sociological Review 64: 113-132.

South, Scott J. and Eric P. Baumer. 2000. "Deciphering Community and Race Effects on Adolescent Premarital Childbearing.” Social Forces 78: 1379-1407.

Suttles, Gerald. 1968. The Social Order of the Slum: Ethnicity and Territory in the Inner City. Chicago: University of Chicago Press.

Whyte, William. 1943. Streetcorner Society. Chicago: University of Chicago Press.

Wikström, P-O and Butterworth D. 2006. Adolescent Crime : Individual Difference and Lifestyles, Willan Publishing.

Wilson, William Julius. 1987. The Truly Disadvantaged: The Inner-City, the Underclass, and Public Policy. Chicago: University of Chicago Press.

Wilson, William Julius. 1996. When Work Disappears: The World of the New Urban Poor. New York: Knopf. 
Winship, Christopher and David J. Harding. 2008. "A General Strategy for the Identification of Age, Period, Cohort Models: A Mechanism Based Approach," Sociological Methods and Research 36(3): 362-401

Young, Alford A, Jr. 2004. The Minds of Marginalized Black Men: Making Sense of Mobility, Opportunity, and Life Chances. Princeton: Princeton University Press.

Zenk S, Schulz AJ, House JS, Benjamin A, Kannan S. 2005b. “Application of CommunityBased Participatory Research in the Design of an Observational Tool: the Neighborhood Observational Checklist," in Methods in Community-Based Participatory Research for Health (Israel BA, Eng E, Schulz AJ, Parker E, eds). San Francisco:Jossey-Bass, 167187.

Zenk, Shannon N, Amy J Schulz; Barbara A Israel; Sherman A James 2005. "Neighborhood Racial Composition, Neighborhood Poverty, and the Spatial Access." American Journal of Public Health 95(4): 660-667. 
Figure 1. Identifying the Effects of Multiple Mechanisms

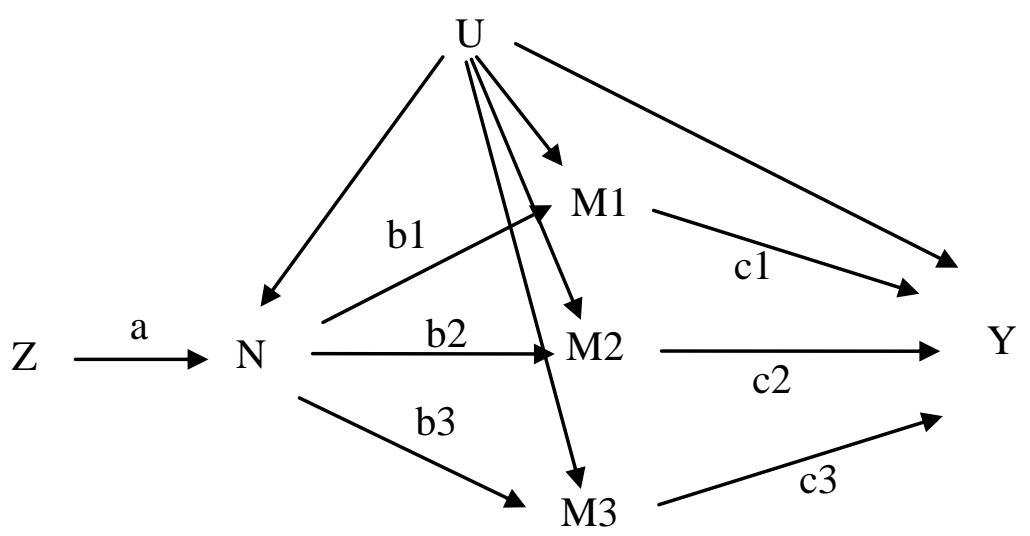




\section{Appendix \\ Unpacking Neighborhood Influences on Education Outcomes: \\ Setting the Stage for Future Research}

\section{Table of Contents}
A. Effect Heterogeneity
B. Further Random Assignment Research Design Considerations

In this web appendix we include a more detailed description of effect heterogeneity and an expanded discussion of our illustrative example introduced in Section V.

\section{A. Effect Heterogeneity}

The theoretical perspectives in Section II of our chapter provide broad outlines of how neighborhood effects on educational outcomes might work, but none of these frameworks adequately considers how the daily experiences of youth differ within neighborhoods, i.e. how they spend their time, where they spend their time, with whom they spend their time, and how such exposure influences attitudes, frames, expectations, etc. These differences are a potentially important but largely uninvestigated source of effect heterogeneity. Here we provide further discussion to motivate the importance of effect heterogeneity in a conceptual framework as well as in the design of studies and subsequent empirical analysis.

We begin with differences across individuals in social networks as one possible source of neighborhood effect heterogeneity. Though most neighborhood effects theories implicitly assume that neighborhoods play some role in structuring the social networks of their residents, we actually know little about whether-or more importantly for whom- 
this is the case, particularly among youth. Social networks are one of the key conduits through which information and cultural frames or scripts are transmitted, but by no means the only one. Social networks of youth of a similar age ("peer networks") have received considerable attention in the literature (e.g. Anderson 1999). Such peer networks may play important roles as cultural conduits, as most theories of peer effects assume, but our theories need to be more specific about who those peers are, which peer attachments are more common among young people in poor neighborhoods, and what is transmitted through peer networks. Harding $(2009,2010)$ argues that older adolescents and young adults on the street in poor violent neighborhoods have considerable cultural power and play an important role in socializing younger adolescents by exposing them to local cultural frames and scripts regarding schooling and sexual behavior.

A second source of effect heterogeneity is different behavioral adaptations to the challenges of daily life in poor neighborhoods. A focus on behavioral adaptations explicitly considers the individual as an actor who can adapt in different ways to mitigate or overcome challenges faced in different neighborhoods. The distinction developed by Sharkey (2006) between "imposed" environments (everything present in the neighborhood where an individual lives) and "selected" environments (the people and institutions with whom he or she interacts) highlights the idea that youth living in the same neighborhood may choose very different social environments for themselves. Different choices or adaptations can have different consequences. For example, violent neighborhoods provide particular challenges to adolescents. In order to feel safe, some adolescents may engage in behaviors such as demonstrating toughness, altering daily travel routines, forming strong bonds of mutual protection with friends, or relying on 
older individuals for protection in order to avoid victimization (Anderson 1999, Harding 2009, 2010). These same behaviors may have unintended educational consequences because they can be interpreted as resistant or disruptive by teachers (Dance 2002). Another example is provided by Carter (2005) who argues that "cultural authenticity" (in the form of speech styles, clothing, music and other tastes) among ethno-racial minority groups can have positive payoffs in terms of group membership and solidarity (what Carter calls "non-dominant cultural capital") but also can be misinterpreted by white middle-class teachers as oppositional or resistant. Parents may also adapt their parenting practices in response to the neighborhood environment, for example by limiting their children's interactions with neighbors (Furstenberg et al. 1999, Jarrett 1997ab).

A third potential source of neighborhood effect heterogeneity is variation in family characteristics and the interaction between family characteristics and the properties of social settings. Here effect heterogeneity is driven less by differences in social interactions and more by differences between individuals and families. We illustrate some of the complexities inherent in this type of effect heterogeneity through the example in Appendix Table 1. This relatively simple example focuses on one type of neighborhood mechanism, a neighborhood resource, and two sources of effect heterogeneity: family capacity and access to non-neighborhood opportunities. 


\section{Appendix Table 1: Effect Heterogeneity by Family Capacity for Neighborhood}

\section{Resource}

\begin{tabular}{|l|c|c|c|c|}
\hline & \multicolumn{2}{|c|}{ High Family Capacity } & \multicolumn{2}{c|}{ Low Family Capacity } \\
\hline & $\begin{array}{c}\text { High Access to } \\
\text { Outside } \\
\text { Opportunities }\end{array}$ & $\begin{array}{c}\text { Low Access to } \\
\text { Outside } \\
\text { Opportunities }\end{array}$ & $\begin{array}{c}\text { High Access to } \\
\text { Outside } \\
\text { Opportunities }\end{array}$ & $\begin{array}{c}\text { Low Access to } \\
\text { Outside } \\
\text { Opportunities }\end{array}$ \\
\hline $\begin{array}{l}\text { High } \\
\text { Neighborhood } \\
\text { Resources }\end{array}$ & + & + & $?$ & - \\
\hline $\begin{array}{l}\text { Low } \\
\text { Neighborhood } \\
\text { Resources }\end{array}$ & + & - & $?$ & - \\
\hline
\end{tabular}

The two rows in the above table represent different types of neighborhoods neighborhoods with either high or low resources. Without loss of generality, neighborhood resources might include different types of social services and non-school educational programs. Examples would be a neighborhood health center, an after-school program, or summer day camp. Such resources can remove youth from the physical and social dangers of the street by providing safe venues as well as positive socialization, for example through well-designed and supervised community centers with an array of ageappropriate social activities. We assume, however, that these resources are not abundant enough to serve all children in the neighborhood. We also assume that such resources might be acquired elsewhere, should an individual or family be able to access non-local resources. Alternatively, this model could be developed to consider how families of different types were affected by neighborhood deficits, e.g. a lack of safe streets.

Presumably, high capacity families would be more capable of minimizing the effects of deficiencies. 
The four columns represent different types of families. Particular differences are discussed below. The cell in each column represents a prediction of whether the outcome on some generic variable of interest would be positive or negative. Examples might be educational attainment, presence of behavioral problems, adolescent pregnancy or gang problems. In this scheme, a neighborhood effect only occurs in a particular column (that is for a particular family type) when the outcome differs depending on whether the neighborhood has a high versus low level of resources.

Our table distinguishes families along two dimensions. First is the capacity of the family. By this we simply mean the ability of a family to take advantage of resources if they are available to them. High capacity families are those that will capitalize on the opportunities available to their children. Low capacity families will not. The level of capacity might be the result of family structure, parental education, family income, the number of children, or parental health.

The second dimension distinguishes families in terms of their access to opportunities outside their neighborhood. These opportunities might have to do with family members or friends. A single mother might send her child each weekend to play with her sister's children in a safer neighborhood. Alternatively, because a neighborhood has good access to public transportation or a family has a car, a parent might well be able to access resources for their children outside the neighborhood that are not available in their own neighborhood, such as a higher quality school.

The above typology results in four types of families: high capacity-high outside opportunities, high capacity-low outside opportunities, low capacity-high outside 
opportunities, low capacity-low outside opportunities families. Now consider why the level of resources in a neighborhood does or does not affect a particular type of family:

High/High $\quad$ Families are able to provide necessary resources for their children whether or not those resources are available in their neighborhood. Example: Wealthy family living in the downtown of a large city whose children attend private schools.

High/Low Because these families have low access to outside opportunities, they only can provide those opportunities to their children if they are available in their neighborhood. Example: A high capacity, but relatively poor family living in a neighborhood with many social services. This is the one family type where neighborhood resources make a difference. Example: A low-income family taking the initiative to enroll their child in a specially tailored school program, like that offered in the Harlem Children's Zone.

Low/High What the prediction should be here is unclear, though the low capacity of the family means that they will not take advantage of neighborhood resources even when they are present. What is unclear is whether the presence of outside opportunities results in a positive outcome. For example, as Carol Stack argued in All Our Kin (1974), the presence of high capacity extended kin members results in positive outcomes for the children of a low capacity parent.

Low/Low These are families that are low capacity and do not have good outside opportunities. Even if their neighborhood has considerable resources, they are unable to take advantage of them. Example: a single parent addicted to drugs who has alienated her extended family.

It is important to remember that this model is meant to represent a set of ideal types.

Obviously, it is quite simplistic. Despite its simplicity, it provides at least two important insights. First, of the four types of families, the resources of the neighborhood only make a difference for one type of family — the high capacity family that does not have good access to opportunities outside the neighborhood. All other families are not affected by neighborhood resources because either they do not need them (they have high access to 
opportunities outside the neighborhood) or they are unable to use them (they are low capacity).

Second, our schema, if correct, would suggest that policy interventions that seek to enhance place-based resources should focus on three aims. First is providing good neighborhood resources to high capacity families. This might mean either investing in their neighborhoods or moving them from a low to a high resource neighborhood. Second, policy interventions should be developed to help low capacity families access the resources in their neighborhoods. This means getting local social service organizations to reach out to the most troubled families as opposed to simply helping those who actively seek assistance. Third, our model demonstrates the appreciable difficulties in improving the lives of low capacity families in low resource neighborhoods. To help these families, one needs to get them access to resources generally and, just as importantly, to help them to take full advantage of those resources.

\section{B. Further Random Assignment Design Research Considerations}

In Section V of our chapter we very briefly sketched a study design to examine one potential neighborhood effects mechanisms, neighborhood violence. The goal of the hypothetical study was to estimate the effects of neighborhood violence on youth educational outcomes. Here we discuss features of the research design in greater detail. Our aim is not to present a complete research design (or even one that is clearly feasible without vast resources), but rather to discuss aspects of the research design that illustrate ways to apply the ideas discussed in the main text. 
Consider first the various approaches to identifying the effect of neighborhood violence. Handled through a standard multivariate regression analysis, any estimate of neighborhood violence on youth educational achievement might be biased by confounding characteristics of the youth, his or her family, and other aspects of the neighborhood environment that influence school performance and that are also correlated with neighborhood violence. This situation leaves researchers in a dilemma: Where can a researcher find exogenous sources of variation to identify the effects of a neighborhood characteristic like exposure to violence? We need one or more variables that produce variation in exposure to violence but do not otherwise affect the outcome of interest. We can think about this by considering two sources of plausible exogenous variation: variation that is planned and variation emerging from a natural experiment. Planned variation can arise from random assignment or experimental designs. Randomization ensures that individuals (or whatever unit is randomized) assigned to the treatment and control groups are the same across both observed and unobserved characteristics, except for chance variation. One strategy is for the researcher to indirectly manipulate neighborhood conditions by encouraging residential mobility, for example, by offering a housing voucher (such as in the Moving to Opportunity mobility experiment). A second strategy is to directly alter neighborhood characteristics through a place-based intervention. In a placed-based intervention, a random subset of neighborhoods would experience a direct intervention such as newly built after school clubs or the addition of street workers to reduce gang violence, and a random subset of otherwise comparable neighborhoods would not receive the intervention. A third strategy, the one we use in this example, is to randomly manipulate exposure to the neighborhood characteristic. 
Well-conceived and properly implemented random assignment studies have high potential for unraveling causal effects, but researchers are faced with trade-offs. Practical and ethical considerations may make the manipulation of certain processes difficult or undesirable no matter how important these processes may be from an explanatory perspective. However, mechanisms that involve, for example, gangs, drugs, or violence can still be examined through experimental manipulations as long as the manipulation focuses on increasing the safety of the treated group and leaves the control group no worse off. Further, a number of circumstances can dilute the intended treatment effects (often described as issues of noncompliance). For example, participants may not take up the offer of a new opportunity, service or program and ethical considerations often preclude requiring or mandating participation. Researchers also cannot prevent study participants from accessing a similar service on their own, or unintentionally receiving the experimental treatment even if initially assigned to the control group. Second, depending on the mechanisms and outcomes of interest, experiments can be expensive and may require a very long follow-up before meaningful outcomes are measurable. The costs of experiments may limit sample sizes and statistical power to detect effects that are small or moderate in magnitude. Third, a series of practical constraints must be carefully weighed: implementation and noncompliance may mean that not everyone in the treatment group receives the intended intervention, the study population may be narrowly defined and thus results may not be generalizable to full-scale or mandatory programs, and well-done experiments can only focus on a limited number of interventions. As a result, while experiments are useful for identifying causal effects once leading hypotheses have been identified, they may not be as useful for generating hypotheses. In fact, the 
power of randomized designs to make contributions to the literature may not be fully leveraged until after researchers have analyzed observational and/or qualitative data to generate a narrow set of hypotheses. For more on random assignment study designs, we refer readers to Orr (1999).

The second type of study design that harnesses exogenous variation in the treatment is a "natural experiment" in which policy manipulations or other social or economic processes not directly related to the outcome create exogenous variation in either residential mobility or neighborhood characteristics. Examples include the residential mobility created by demolition of public housing projects (Jacob 2004), and the use of natural boundaries that determine public school districts (Hoxby 2001). Here researchers must be opportunistic and leverage naturally occurring exogenous variation in the neighborhoods that youth experience. The challenge is that such variation must be known a priori and must be measured. Moreover, any claim that such variation is truly exogenous is based on assumptions about social and economic processes and subject to skepticism from other researchers. The neighborhood effects literature has produced few if any natural experiments whose identification strategies have not been met by significant skepticism.

In the illustrative example in Section V of the chapter, we focus on planned variation through a random assignment design. We propose manipulating the amount of time youth spend in their neighborhood under the assumption that out-of-school time use affects exposure to violence which affects educational performance. Note that there are multiple ways in which exposure to violence may affect educational outcomes (see Section II in the main text) and that many, such as stress or trauma, require long-term 
cumulative exposure to violence. Since in this example the change in exposure to violence is short term, we focus on outcomes that are more likely to respond immediately to a reduction in exposure to violence, such as school attendance, tardiness, and engagement in school. These responses to exposure to violence can ultimately contribute to achievement.

Our proposed study design is individual random assignment embedded within a matched-neighborhood design. The matched neighborhoods allow us to place youth into neighborhoods that are similar to their own on demographic and economic characteristics but have lower levels of violence. In the first stage, selected high violence neighborhoods are matched to neighborhoods comparable on poverty, race, and education that have lower rates of violence (see Seith et al, 2003 on the feasibility of matched-neighborhood designs). An intervention could be designed that is non-academic and that involves engagement with the neighborhood - such as working on a local clothing drive. For the second stage of the study, we could recruit youth from the neighborhoods with higher levels of neighborhood violence, so that the neighborhoods where subjects are assigned by the intervention are all less risky than their home neighborhoods. We would randomly assign these youth to teams in different locations outside of their own neighborhoods, some of which would have lower neighborhood violence than others. Some type of financial incentive might be designed to encourage participation in the intervention activity.

The first important piece of data collection is baseline information through a survey measuring the characteristics of the individuals and their families prior to the intervention (including a variety of indicators to gauge family capacity as described in the 
first section of this appendix) and baseline characteristics of the neighborhoods through census or comparable community-level data including crime rates. During the intervention, monitoring of program implementation verifies treatment fidelity and identifies any unintended consequences. To assess the impact of the intervention, data collection would include a follow-up survey to document youth time use (hours spent engaged in certain activities, where and with whom); longitudinal school records to track youth attendance, disciplinary actions taken by the school, and school grades over the course of the intervention; follow-up information on the characteristics of the experimental and control neighborhoods; and open ended qualitative interviews to capture more nuanced aspects of time use and participation in the program as well as subjects' experiences with schooling and with neighborhood violence both prior to and during the intervention.

Our proposed randomized design to manipulate time use can test the hypothesis that spending time in a lower violence neighborhood results in lower exposure to violence and increases youth school attendance, reduces tardiness and school disciplinary action. Under this hypothesis, we would expect, for example, the difference between post-intervention attendance rates and pre-intervention attendance rates to be larger in groups assigned to lower violence neighborhoods. The intervention manipulates exposure to neighborhoods that differ in their levels of violence but does not directly manipulate exposure to violence itself. We can use survey data and qualitative data to measure the intervention's impact on exposure by asking youth about their experiences with violence in their day-to-day activities and traveling to and from school, and we can consider measures of anxiety or stress that can be captured through survey reports. 
Stratification of the sample based on pre-intervention youth characteristics - such as measures of their vulnerability - or family characteristics -- such as family capacity -could be used to explicitly test hypotheses about effect heterogeneity. For example, we might expect that only highly vulnerable youth in a high violence neighborhood would be affected by exposure to violence. The sample would be stratified by youth vulnerability and then the youth in each stratum would be randomized to the control or intervention group.

Although this research design is appealing in its simplicity, it is important to note its limitations. The randomized experiment will not allow for testing of other neighborhood effects mechanisms unless one is willing to make very strong assumptions about selection on observables and independent mechanisms. As we discuss in the main text, examining multiple mechanisms requires multiple sources of exogenous variation. Moreover, even well-thought out simple designs can generate perplexing findings that might be due to the unanticipated effects of treatment assignment, and this sometimes necessitates a reconsideration of the initial study design. Nevertheless, when designed well, randomized experiments and natural experiments, such as the one described here focused on neighborhood violence, offer promise for uncovering mechanisms. 


\section{Appendix References}

Anderson, Elijah. 1999. Code of the Street: Decency, Violence, and the Moral Life of the Inner-city. New York: W. W. Norton.

Angrist, Joshua, Guido Imbens, and Don Rubin. 1996. Identification of Causal Effects Using Instrumental Variables. Journal of American Statistical Association 91(343): 444-55.

Brooks-Gunn, Jeanne, Greg J. Duncan, Pamela K. Klebanov, and Naomi Sealand. 1993. "Do Neighborhoods Influence Child and Adolescent Development?" American Journal of Sociology 99:353-95.

Carter, Prudence L. 2005. Keepin' It Real: School Success Beyond Black and White. New York: Oxford University Press.

Dance, L. Janelle. 2002. Tough Fronts: The Impact of Street Culture on Schooling. New York: Routledge Falmer Press.

Furstenburg, Frank F. et al (eds). 1999. Managing to Make It: Urban Families and Adolescent Success. Chicago: University of Chicago Press.

Grannis, Rick. 1998. "The Importance of Trivial Streets: Residential Streets and Residential Segregation.” American Journal of Sociology 103(6): 1530-64.

Harding, David J. 2009. "Violence, Older Peers, and the Socialization of Adolescent Boys in Disadvantaged Neighborhoods." American Sociological Review 74(3): 445-464.

Harding, David J. 2010. Living the Drama: Community, Conflict, and Culture Among Inner-City Boys. Chicago: University of Chicago Press.

Hoxby, C. M. 2001. “All School Finance Equalizations Are Not Created Equal.” The Quarterly Journal of Economics, 116, 1189-1230.

Jacob, Brian 2004. "Public Housing, Housing Vouchers and Student Achievement: Evidence from Public Housing Demolitions in Chicago." American Economic Review. 94(1): 233-258.

Jarrett, Robin L. 1997a. "African American Family and Parenting Strategies in Impoverished Neighborhoods," Qualitative Sociology 20: 275-288.

Jarrett, Robin L. 1997b. "Bringing Families Back In: Neighborhood Effects on Child Development.” Pp. 48-64 in Jeanne Brooks-Gunn, Greg J. Duncan, and J. 
Lawrence Aber, eds. Neighborhood Poverty, Vol. 2: Policy Implications in Studying Neighborhoods. New York: Russell Sage

Krieger N, Chen JT, Waterman PD, Soobader MJ, Subramanian SV, Carson R. 2002. "Geocoding and Monitoring of US Socioeconomic Inequalities in Mortality and Cancer Incidence: Does the Choice of Area-Based Measure and Geographic Level Matter?: the Public Health Disparities Geocoding Project." American Journal of Epidemiology. 156:471-482.

Orr, Larry L. (1999). Social Experiments: Evaluating Public Programs with Experimental Methods. Thousand Oaks, CA: Sage Publications.

Sampson, Robert J. and Steve Raudenbush. 1999. "Systematic Social Observation of Public Spaces: A New Look at Disorder in Urban Neighborhoods." American Journal of Sociology 105: 603-651.

Seith, David C., Verma, Nandita, Bloom, Howard S., and George C. Galster. (2003). Exploring the Feasibility and Quality of Matched Neighborhood Research Designs. MDRC.

Sharkey, Patrick T. 2006. "Navigating Dangerous Streets: The Sources and Consequences of Street Efficacy." American Sociological Review 71: 826-846.

Stack, Carol. 1974. All Our Kin: Strategies for Survival in a Black Community. New York: Harper \& Row. 